\title{
INTRODUCTION
}

\section{P.M. Calverley}

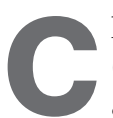

hronic obstructive pulmonary disease (COPD) is characterised by inflammation, airflow limitation that is not fully reversible and a gradual loss of lung function [1]. The most recent COPD guidelines from the Global Initiative on Chronic Obstructive Lung Disease (GOLD) emphasise the key point that an abnormal inflammatory response in the lungs to noxious gases or particles is central to the pathology of COPD [2]. The association of a systemic cellular and molecular inflammatory response with COPD is also well documented. Recent literature provide evidence of elevations in multiple serum markers of inflammation in patients with COPD, including interleukins, tumour necrosis factor- $\alpha$ and C-reactive protein $[3,4]$. This systemic inflammation may contribute to the wide range of serious comorbidities observed in patients with COPD, including increased risk for cardiovascular disease, osteoporosis, diabetes, gastrointestinal diseases, changes in body composition (loss of lean muscle mass) and anaemia [4].

The central role of inflammatory processes in the pulmonary and, perhaps, the systemic comorbidities of COPD have focused attention on new treatments for this disease that directly target these inflammatory processes. New therapies being evaluated for the treatment of COPD include agents directed against the influx of inflammatory cells into the airways and lung parenchyma that occurs in COPD, and broadspectrum anti-inflammatory drugs, such as inhibitors of phosphodiesterase (PDE)4, p38 mitogenactivated protein kinase, nuclear factor- $\kappa \mathrm{B}$ and phosphoinositide-3 kinase- $\gamma$. Other more specific approaches include antioxidants, inhibitors of inducible nitric oxide synthase and leukotriene $\mathrm{B}_{4}$ receptor antagonists [5-7].

At present, smoking cessation remains the most important intervention for modifying the course of COPD [8]; there is little evidence that other current therapies for COPD have the potential to slow or halt the progression of this disease. However, recent results from the Towards a Revolution in COPD Health (TORCH) study have suggested that treatment with a combination of fluticasone propionate and salmeterol decreased the risk for death among patients with COPD by $18 \%$ versus placebo $(12.6 \%$ for active treatment versus $15.2 \%$ for placebo; absolute risk reduction $2.6 \%$ ) [9]. It is hoped that newer targeted therapies can produce more substantial increases in survival as well as significant improvements in pulmonary function in patients with COPD.

The three articles comprising the symposium presented herein focus on inflammatory processes in COPD and the degree to which they can be modified by current and emerging therapies for this disease. RENNARD [10] focuses on chronic inflammation and injury of both the airways and the parenchymal pulmonary structures in patients with COPD, and discusses the repair processes stimulated by these inflammatory events. Results summarised in the article by RENNARD [10] suggest that complex relationships among a large number of mediators determine whether or not repair leads to restoration of normal tissue structure and function or pathological alterations that further impair pulmonary function in COPD patients.

The article by RocHE [11] considers disease progression in patients with COPD and the possibility that currently approved treatments may have the potential to slow or arrest progressive decline in lung function, and perhaps even decrease mortality in patients with COPD. Important studies considered in detail in the article by RocHE [11] include the long-term trial of tiotropium in patients with COPD [12] and TORCH [9]. ROCHE [10] also emphasises the importance of early diagnosis and intervention to maximise the potential of treatment in order to slow disease progression in COPD patients.

The article by McIvor [13] reviews new COPD therapies, emphasising PDE4 inhibitors that are being developed for the treatment of patients with this disease. Pre-clinical results for these agents have demonstrated their potential to blunt both the inflammatory processes and pulmonary remodelling characteristic of COPD. Clinical results for one agent in this class, roflumilast, have further demonstrated that PDE4 inhibition has the potential to safely improve pulmonary function and decrease the frequency of exacerbations among patients with a wide range of COPD severities. The fact that roflumilast is administered orally and is systemically bioavailable raises the possibility that this PDE4 inhibitor may also benefit the systemic comorbidities commonly observed in patients with COPD.
CORRESPONDENCE

P.M. Calverley

School of Clinical Sciences University Hospital Aintree University of Liverpool Liverpool L9 7AL UK Fax: 441515295888 E-mail: pmacal@liverpool.ac.uk

\section{STATEMENT OF INTEREST}

P.M. Calverley has received honoraria for speaking at symposia supported by Altana (Nycomed) and his department has received research funds to support clinical trials of roflumilast. 
In summary, the results and discussion presented in this issue of the European Respiratory Review provide the reader with a concise update on chronic obstructive pulmonary disease pathophysiology, the latest data on currently approved therapies, and new information on emerging treatments that may greatly benefit patients with this disease.

\section{REFERENCES}

1 Rodriguez-Roisin R. The airway pathophysiology of COPD: implications for treatment. COPD 2005; 2: 253-262.

2 Global Strategy for Diagnosis, Management, and Prevention of COPD. 2006. www.goldcopd.com/Guideline List.asp?11 $=2 \& 12=1$. Date last updated: November 2006. Date last accessed: June 18, 2007.

3 Yende S, Waterer GW, Tolley EA, et al. Inflammatory markers are associated with ventilatory limitation and muscle dysfunction in obstructive lung disease in well functioning elderly subjects. Thorax 2006; 61: 10-16.

4 Sevenoaks MJ, Stockley RA. Chronic obstructive pulmonary disease, inflammation and co-morbidity - a common inflammatory phenotype? Respir Res 2006; 7: 70.

5 Barnes PJ. ABC of chronic obstructive pulmonary disease. Future treatments. BMJ 2006; 333: 246-248.
6 Barnes PJ, Stockley RA. COPD: current therapeutic interventions and future approaches. Eur Respir J 2005; 25: 1084-1106.

7 Barnes PJ, Hansel TT. Prospects for new drugs for chronic obstructive pulmonary disease. Lancet 2004; 364: 985-996.

8 Anthonisen NR, Skeans MA, Wise RA, et al. The effects of a smoking cessation intervention on 14.5-year mortality: a randomized clinical trial. Ann Intern Med. 2005; 142 233-239.

9 Calverley PM, Celli B, Anderson JA, et al. Salmeterol and fluticasone propionate and survival in chronic obstructive pulmonary disease. N Engl J Med 2007; 356: 775-789.

10 Rennard SI. Inflammation in COPD: a link to systemic comorbidities. Eur Respir Rev 2007; 16: 91-97.

11 Roche N. Where current pharmacological therapies fall short in COPD: symptom control is not enough. Eur Respir Rev 2007; 16: 98-104.

12 Anzueto A, Tashkin D, Menjoge S, Kesten S. One-year analysis of longitudinal changes in spirometry in patients with COPD receiving tiotropium. Pulm Pharmacol Ther 2005; 18: 75-81.

13 McIvor RA. Future options for disease intervention: important advances in phosphodiesterase 4 inhibitors. Eur Respir Rev 2007; 16: 105-112. 